\title{
One flerovium atom at a time
}

\author{
The chemistry of element 114 seems to be in reach, yet Peter Schwerdtfeger cautions that we should \\ expect the unexpected from this young element, which is so different to its lighter counterparts.
}

T he last primordial element in the periodic table - with a lifetime comparable to the age of our Earth is plutonium, which is found only in trace amounts in a mineral named bastnasite. All elements beyond ${ }_{94} \mathrm{Pu}$ have been produced in nuclear fusion reactions in a few laboratories around the world, most recently from an ongoing Dubna-Livermore collaboration that led in 2004 to the discovery of flerovium.

Four isotopes of element 114, with atomic numbers from 286 to 289 , have been produced at a heavy-ion cyclotron from nuclear fusion reactions between ${ }^{48} \mathrm{Ca}$ ion beams and targets made of several isotopes of plutonium or curium. In 2011, the International Union of Pure and Applied Chemistry agreed to name element 114 after the Flerov Laboratory of Nuclear Reactions in Dubna, which itself took the name of its founder, Georgii Nikolajevich Flerov (pictured), a prominent Russian nuclear physicist and co-discoverer of spontaneous nuclear fission. Flerovium takes its place in the periodic table as the last member of group 14, which begins with carbon and until recently ended with lead.

This exotic superheavy element has such a high nuclear charge that it can only be produced one-atom-at-a-time by fusion over several months - a rather long period of time compared with its nuclear decay, which is in the range of seconds. Flerovium is exciting in several aspects; in particular, it may help to understand nuclear matter in the high proton limit, and in the search for the island of stability, with isotopes of high nuclear stability in a certain proton and neutron range.

The atomic nucleus consists of proton and neutron shells, very much like the electronic shells we are all familiar with in chemistry. A completely filled shell would have a 'magic number' of neutrons and protons, which would confer particular

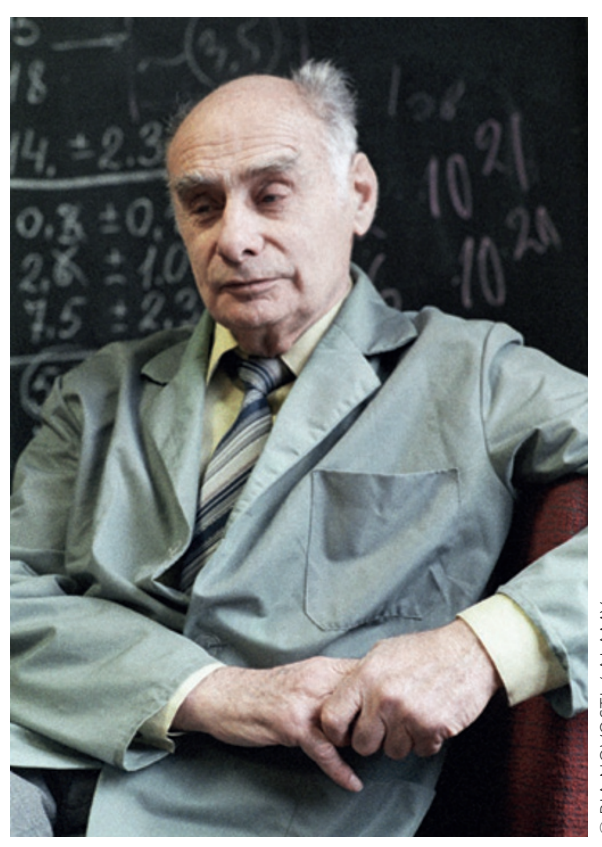

stability to the element. For more than four decades, shell closure has been predicted at $Z=114$ for protons and at $N=184$ for neutrons. However, although most scientists have long agreed on the number for neutron shell closure, that for proton shell closure depends very much on the model used to determine the underlying nuclear structure.

Unlike for the electronic structure, the strong interactions between the nucleons are difficult to model accurately, and shell closure has also been predicted at $Z=120$, 122 or even 126. Accurate measurements of the radioactive decay properties of superheavy elements are therefore required to refine these nuclear models. The most neutron-rich isotope of flerovium known at present is ${ }^{289} \mathrm{Fl}$ with 114 protons and 175 neutrons, but it still falls short of neutron shell closure by 9 neutrons and it is less than clear how to get them inside the nucleus.

Nuclear decay times in the range of seconds are sufficient to perform atom-ata-time chemistry on superheavy elements, but the design of such experiments at the cutting edge of current chemical technology requires some knowledge of their chemical behaviour. The use of modern relativistic quantum chemical methods that deal with the Dirac instead of the Schrödinger equation offers an ideal tool for probing flerovium's reactivity.

It was the late Kenneth Pitzer who pointed out as early as 1975 that strong relativistic effects could lead to an electronic shell closure in element 114. Large spinorbit coupling effects sufficiently separate the $7 p_{1 / 2}$ from the $7 p_{3 / 2}$ shells (by more than $\left.300 \mathrm{~kJ} \mathrm{~mol}^{-1}\right)$, and flerovium adopts a $7 s^{2}\left(7 p_{1 / 2}\right)^{2}$ closed-shell configuration. It is therefore predicted to be rather volatile and chemically inert. Pitzer even went as far as to suggest that element 114 could be a gas at room temperature in contrast to lead or tin, its lighter counterparts in group 14.

It is, however, not always possible to predict bulk phenomena from simple atomic properties. Recent solid-state calculations in our group gave some surprising results. Atoms in bulk metallic flerovium are only weakly bound, less than in mercury, but more than in xenon (cohesive energies of 50, 75 and $16 \mathrm{~kJ} \mathrm{~mol}^{-1}$, respectively). This suggests that flerovium would be a metallic liquid at room temperature, much like mercury. Moreover the $7 s$ orbitals, stabilized by relativistic effects, may become chemically inert.

Heinz Gäggeler and co-workers have probed the adsorption of ${ }_{112} \mathrm{Cn}$ and ${ }_{114} \mathrm{Fl}$ on gold surfaces. So far, only three events have been seen that can be assigned to flerovium atoms, suggesting that element 114 behaves more like a noble, yet still metallic, gas. However, the results are not yet conclusive and confirmation experiments are ongoing. What kind of chemistry can flerovium engage in? Let us expect the unexpected!

PETER SCHWERDTFEGER is at the New Zealand Institute for Advanced Studies (NZIAS) at Massey University in Auckland, New Zealand.

e-mail: peter.schwerdtfeger@gmail.com 